Supporting Information

\title{
Pressure-Driven and Creep-Enabled Interface Evolution in Sodium Metal Batteries
}

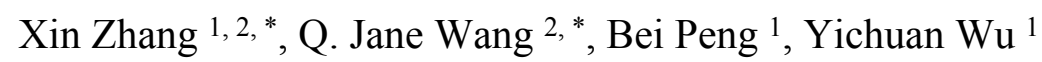

1. School of Mechanical and Electrical Engineering, University of Electronic Science and Technology of China, Chengdu 611731, China

2. Department of Mechanical Engineering, Northwestern University, Evanston, IL 60208

* Corresponding Authors: Q. Jane Wang, qwang@northwestern.edu,

Xin Zhang, zhangxin@uestc.edu.cn

This file includes:

Supporting Figures S1-S4 
In Figure S1, the gap, $g_{N a-S E}$, between the solid electrolyte and the sodium metal is calculated by considering the $\mathrm{Na}$ elastoplastic deformation, the roughness of both surfaces, $\mathrm{Na}$ creep, and $\mathrm{Na}$ plating/stripping,

$$
\begin{aligned}
g_{N a-S E}(x, y)=- & u_{N a}(x, y)+s_{\text {equiv }}(x, y) \\
& \quad h_{\text {creep }}(x, y)+\epsilon \cdot h_{p / s}(x, y)-\delta_{N a-S E},
\end{aligned}
$$

where the depth-direction elastoplastic deformation of the Na surface, $u_{N a}(x, y)$, can be calculated by

$$
u_{N a}(x, y)=\frac{2}{\pi E^{\prime}} \iint \frac{p(m, n)}{\sqrt{(x-m)^{2}+(y-n)^{2}}} d m d n
$$

The rough surface topography, $s_{\text {equiv }}(x, y)$, is shown in Figures S1(a) and (b). The creep deformation, $h_{\text {creep }}(x, y)$, is given in Eq. (12), and the height change due to stripping/plating, $h_{p / s}(x, y)$, is given in Eq. (13).

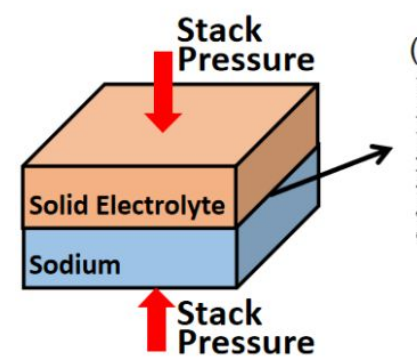

(a)

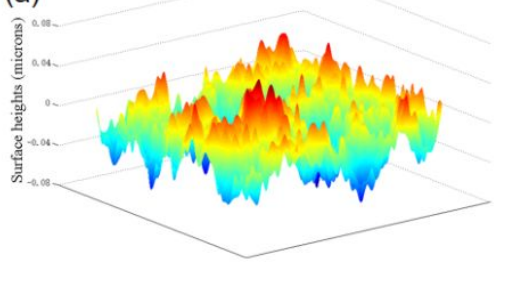

(b)

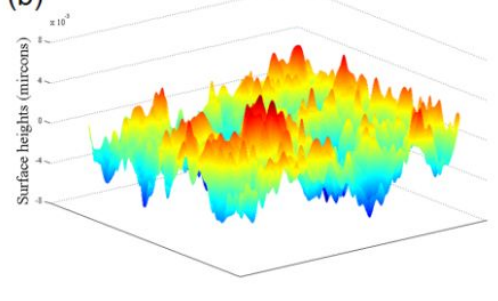

Figure S1 Contact model for the interface of solid electrolyte and sodium metal, subjected to stack pressure. (a) and (b) are topographical plots of rough surfaces using in calculations.

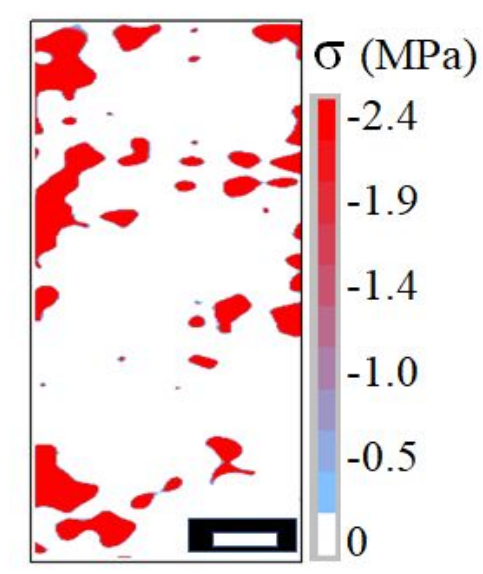

Figure S2 Contact stress map at stack pressure of $0.5 \mathrm{MPa}$, showing about $18 \%$ contact 
fraction. The scale bar represents 40 microns. The Na hardness is $2.4 \mathrm{MPa}$.

(a)

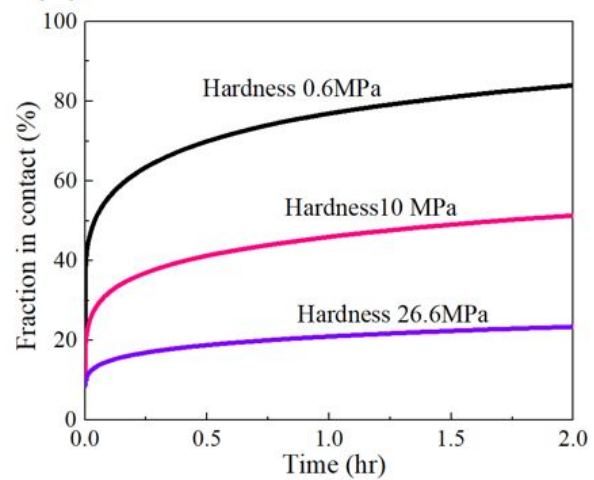

(b)

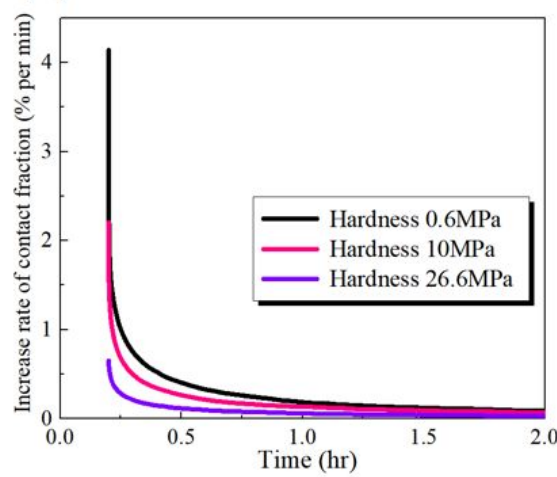

Figure S3 (a) Contact fraction as a function of time for various $\mathrm{Na}$ hardnesses under the stack pressure of $0.5 \mathrm{MPa}$; (b) Rate of increase in contact fraction as a function of time for various $\mathrm{Na}$ hardness under the stack pressure of $0.5 \mathrm{MPa}$.

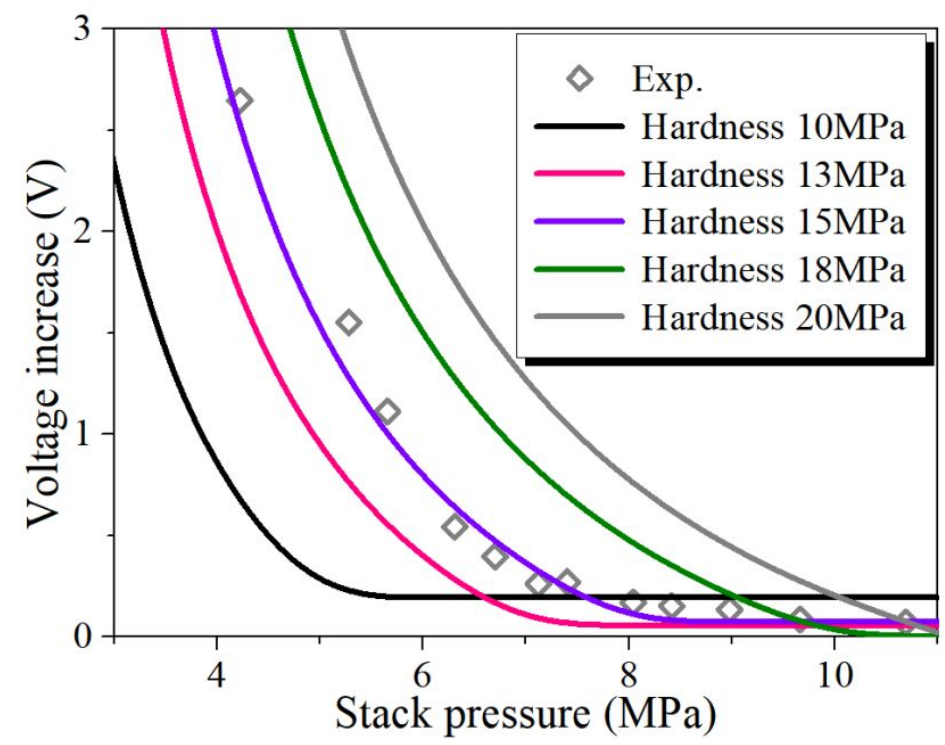

Figure S4 Voltage increase as functions of stack pressures for various Na hardness in a stripping process. The experimental data is from Jolly et al. ${ }^{1}$.

\section{References}

(1) Jolly, D. S.; Ning, Z.; Darnbrough, J. E.; Kasemchainan, J.; Hartley, G. O.; Adamson, P.; Armstrong, D. E.; Marrow, J.; Bruce, P. G. Sodium/Na $\beta^{\prime \prime}$ Alumina Interface: Effect of Pressure on Voids. ACS Appl. Mater. Interfaces 2019, 12(1), 678685. 